\title{
A relative radiometric correction method based on geographically weighted regression model
}

\author{
Chengfeng LUO \\ Institute of Photogrammetry and Remote Sensing, \\ Chinese Academy of Surveying and Mapping, \\ Beijing 100830, China \\ chfluo@casm.ac.cn
}

Haoyan YOU

School of Geometrics, Liaoning Technical University, Fuxin, 123000, China

youhaoyan1988@126.com

\author{
Weilong WU \\ Faculty of Information Engineering, China University of \\ Geosciences, WuHan, 430000, China \\ weilong11307105@163.com
}

\author{
Jiao WANG \\ College of Geomatics, Shandong University of Science and \\ Technology, 266590, China \\ majestic5@126.com
}

\begin{abstract}
- to detect the change in the terrain using multitemporal images has becoming one of the important applications of remote sensing technology. In order to receive a result with high accuracy, the relative radiometric correction among images must be done before the detection. The surface radiation is affected by spatial correlation among the surface objects. This study introduced the geographically weighted regression to the radiometric correction process, and proposed a radiometric correction method based on the geographically weighted regression model. The method includes three main steps. Firstly, iterative weighted multivariate change detection is used to select the invariable pixels as samples. Secondly, radiation correction linear model is built at each sampling point based on geographically weighted regression. Finally the radiometric correction values of target points are calculated with the model of closest point. In the test, using the proposed method a good visual effect can be received. And the precision evaluation indexes are better than those of results from the orthogonal regression radiometric correction. Especially, the information entropy index is almost as twice much as the original image and that of orthogonal regression radiometric correction. It can be concluded that the proposed method in this paper can ensure the radiometric correction visual effect and enhance the details of performance ability of images at the same time.
\end{abstract}

Key words: relative radiometric correction, geographically weighted regression (GWR), information entropy, invariant features points

\section{INTRODUCTION}

Because of different atmospheric conditions, illumination conditions, surface relief, soil moisture, vegetation climate, sensor imaging model and the side view of imaging ${ }^{[1,2]}$, the radiation difference is obvious for the remote sensing images in the same area. In order to extract the change information from multi-temporal remote sensing images more accurately, it is necessary to eliminate the radiation difference. During the

This paper supported by the national science fund of China (No:40901228), and the basic scientific research fund of CASM (No: 7771301). processing of relative radiometric correction, a reference image is selected firstly, and the radiometric values in other images from different times are matched to the referent one. These steps can reduce the effect from the sensor, atmospheric conditions and solar radiation ${ }^{[3,4]}$. Compared with absolute radiometric correction, this way is easy because a variety of related parameters are not required to calibrate the effects of the sensor and atmospheric conditions ${ }^{[3-5]}$.

There are three ways to realize relative radiometric correction, including statistical method, most dark target method ${ }^{[6]}$ and statistical regression method. Several methods are used widely such as image regression(IR), pseudo-invariant features $(\mathrm{PIF})^{[9]}$, dark set-bright set normalization(DB), no change set radiometric normalization(NC), histogram matching(HM), MAD method etc. The accuracy of radiometric correction with statistical regression method is relatively high ${ }^{[7-9]}$. Such methods have a common understanding that the response of detector radiance is linear. But the response of optical remote sensor to surface material is nonlinear. This had been recognized by some researches ${ }^{[10-12]}$. For the relative radiometric correction, the result from model with unified correction coefficient is not good in the range of nonlinear response.

Relative radiometric correction of remote sensing data is affected by the characteristics of natural landscape factors. It is also one of the reasons causing optical remote sensor nonlinear response problem. The adjacent relationship of surface objects induces their radiation with spatial correlation characteristics. The GWR method was introduced into radiometric correction processing in the study. The GWR brings the spatial haracteristics of ground objects radiation into the traditional OLS model. To solve the spatial non-stationarity of the model it calculates the parameter of regression model for every local point which improves the accuracy of radiometric correction greatly. 


\section{METHOD}

\section{A. The principle of $G W R$}

GWR model is a new method proposed by British Fotheringham during the study of space stability. It allows local nonparametric estimation. To reflect the influence of geographical position, samples with different geographical positions have different parameters. It is consistent with the fact $^{[13]}$.

GWR brings the spatial features of objects into the regression model to analysis, and calculates a local equation at each point. The contribution of the position is measured by weighting function of the space adjacent degree to the regression point. GWR model can be expressed as follows:

$$
y_{i}=\beta_{0}\left(u_{i}+v_{i}\right)+\sum_{k=1}^{p} \beta_{k}\left(u_{i}+v_{i}\right) x_{i k}+\varepsilon_{i}, i=1,2, \cdots, n
$$

Where $\left(y_{i} ; x_{i 1}, x_{i 2}, \cdots, x_{i p}\right)$ is the observation value of dependent variable ${ }^{y}$ and independent variable ${ }^{x_{1}, x_{2}, \cdots, x_{p}}$ in the

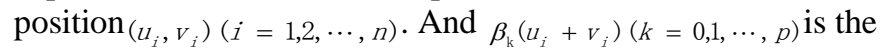
ith observation point with unknown parameter in the position ${ }^{\left(u_{i}, v_{i}\right)}$, and it is arbitrary function $\mathrm{f}^{\left(u_{i}, v_{i}\right)} \cdot \varepsilon_{i}(i=1,2, \cdots, n)$ is the error independent and identically distributed, and is assumed to obey ${ }^{N\left(0, \sigma^{2}\right)}$ generally. According to Tobler's first law of geography, the points closer to point ${ }^{i}$ have the greater influence to the estimation of parameters on the point ${ }^{i}$. It assumes that the regression coefficient of the linear regression model is an arbitrary function of observation point location. The spatial character of the data is taken into consider by the model. Here the weighted least square is adopted to estimate the regression coefficients, and the detailed method can refer document [14].

For every point, there is a local equation calculated by the model. And the weight of the observation value is no longer remaining constant during the regression process. The weight varies with the proximity position to point $i$ :

$$
\hat{\beta}\left(u_{i}, v_{i}\right)=\left(X^{T} W\left(u_{i}, v_{i}\right) X\right)^{-1} X^{T} W\left(u_{i}, v_{i}\right) Y
$$

Where $\hat{\beta}$ is the estimated value of $\beta$, an independent variable matrix, and the first row elements is $1 ; X$ is the independent variable matrix about model factors; $Y$ is the vector of the dependent variable, the matrix about the radiation from the referred imagery; $w$ is a square matrix of weights related to the position of $\left(u_{i}, v_{i}\right)$ in the study area; In the practical operation process, one of the distances, Gauss distance, exponential distance and tricube distance, is used to calculate the spatial weights matrices.

Here Gauss distance is adopted to determine the weight:

$$
W\left(u_{i}, v_{i}\right)=e^{-\frac{1}{2}\left(\frac{d_{\left(u_{i}, v_{i}\right)}}{b}\right)^{2}}
$$

Where $W\left(u_{i}, v_{i}\right)$ is the geographical weight of the ith observation in the dataset related to the $\operatorname{location}_{\left(u_{i}, v_{i}\right)}, d_{\left(u_{i}, v_{i}\right)}$ is the distance between the ith observation and the location $\left(u_{i}, v_{i}\right)$, and $b$ is a quantity known as the bandwidth.

Bandwidth has great influence on the operation results of GWR model. It can be seen as a smoothing parameter, the bigger the smoother. As the bandwidth becomes larger than the weights approach unity, the local GWR model approaches the global OLS model.

\section{$B$. The relative radiometric correction method based on GWR}

The relative radiometric correction method based on GWR need invariant features points as samples. In the study, iterative weighted multivariate change detection was used to select unchanged pixels as samples. The threshold was set to extract the invariant features of multi-variety change detection, and the radiometric correction result is better than traditional artificial selection of invariant features ${ }^{[15]}$.

With these samples, the geographically weighted regression was used to establish spatial correlation model. And each sample point has a fitting equation. When it comes to deal with the whole image, find the closest point of the target point among the invariant samples firstly, then calculate the output value for the target point with the sample's fitting equation. When there are large numbers of invariant features points, the block index method can be considered to improve the computational efficiency.

\section{Accuracy evaluation of relative radiometric correction}

Visual effect is the primary consideration to evaluate the results of the relative radiation correction. In addition, the enhancement of image spatial information and spectral information is another two important aspects. And specific evaluation index includes: the variation coefficient ${ }^{[15]}$, coefficient of correlation, information entropy, deviation index [16].

The variation coefficient, the ratio of the standard deviation and average value, can be used to compare the statistical characteristics parameters of correction image band dispersion.

Deviation Index is used to express the degree of deviation from the image to original image after radiometric correction. The calculation formula is as follows:

$$
\mathrm{D}_{\text {index }}=\frac{1}{M \times N} \sum_{i=0}^{M-1} \sum_{j=0}^{N-1}\left|\frac{I(i, j)-I^{\prime}(i, j)}{I(i, j)}\right| \text { (4) }
$$

In which, $\mathrm{D}_{\text {index }}$ is deviation index, $\mathrm{M} 、 \mathrm{~N}$ is columns and lines of the image, $I(i, j)$ and $I^{\prime}(i, j)$ is radiometric values of $(i, j)$ before and after correction.

Information Entropy reflects the amount of information that image carried. Bigger entropy value means more amount of information carried by the image. If the image gray levels in all probability tend to be equal, the amount of information tends to the maximum. According to Shannon's information theory, information entropy of a 8bit gray image can be calculated as following:

$$
H(x)=-\sum_{i=0}^{255} p_{i} \log _{2} p_{i}
$$

In which, $p_{i}$ is the occurrence probability of value $\mathrm{i}$. 
Correlation coefficient is a quantity value to descript the approximation degree between images. Larger correlation coefficient means closer correlationship.

$$
r=\frac{\sum_{i=1}^{n}\left(x_{i}-\bar{x}\right)\left(x_{i}-\bar{Y}\right)}{\sqrt{\sum_{i=1}^{n}\left(x_{i}-\bar{x}\right)^{2} \sum_{i=1}^{n}\left(y_{i}-\bar{Y}\right)^{2}}}
$$

Here $\mathrm{r}$ is correlation coefficient, $X_{i}$ and $Y_{i}$ are radiometric value of two images, $\bar{X}$ and $\bar{Y}$ are radiometric mean value.

\section{TEST}

\section{A. Test data}

The test data is $\mathrm{HJ}-1 \mathrm{~A} / \mathrm{B}$ data, resolution $30 \mathrm{~m}$, on August 30, 2009 and August 27, 2011. Geometric correction and the atmospheric correction with 6S model had been finished before the correcting. Three bands, R, G, B, with wavelength $0.63 \sim 0.69,0.52 \sim 0.60,0.43 \sim 0.52$ respectively, were used. The data size for test is $5088 \times 4408$.

\section{B. Radiometric correction}

In this test the reference image is data in 2011, the target data in 2009(as shown in figure 1(a)、(b) ). Total 70090 samples, about $0.3 \%$ of all pixels (as shown in figure 1(c)), were selected with iterative weighted multivariate change detection. Before correction the correlation coefficients between these samples are: 0.9979, 0.9947, and 0.9948 in R, G, and B bands respectively. And the correlation coefficient is 0.9999 after these samples were calculated with the proposed method.

After spatial correlation model of these samples were established with geographically weighted regression method, a sample point of each typical features in the image were selected and their fitting equation coefficients are shown in table 1. It is seen from the table their coefficients are different. Although the difference is very small, for radiation values with range between $0-1$, it can't be ignored on the radiation value change.

TABLE I. THE FITTING EQUATION COEFFICIENT FOR DIFFERENT SAMPLE

\begin{tabular}{|c|c|c|}
\hline Band & Vegetation & Bare Land \\
\hline$R$ & $y=1.136 x+0.031$ & $y=1.215 x+0.028$ \\
\hline$G$ & $y=1.079 x+0.017$ & $y=1.251 x+0.002$ \\
\hline$B$ & $y=1.150 x+0.027$ & $y=1.168 x+0.026$ \\
\hline Band & Urban Land & Glacier \\
\hline$R$ & $y=1.165 x+0.031$ & $y=1.195 x+0.029$ \\
\hline$G$ & $y=1.167 x+0.013$ & $y=1.209 x+0.007$ \\
\hline$B$ & $y=1.129 x+0.030$ & $y=1.201 x+0.026$ \\
\hline Band & Water & the orthogonal regression \\
\hline$R$ & $y=1.218 x+0.028$ & $y=1.217 x+0.028$ \\
\hline$G$ & $y=1.247 x+0.003$ & $y=1.223 x+0.007$ \\
\hline$B$ & $y=1.207 x+0.023$ & $y=1.152 x+0.029$ \\
\hline
\end{tabular}

\section{Results}

The correction results with the method proposed in the study were compared with that of the orthogonal regression correction. Here the orthogonal regression method ${ }^{\text {[1] }}$ was adopted to determine the linear relationship between images.

When it comes to the visual effect, there is not obvious difference between the results from GWR and that from orthogonal regression. At the same time, to correct the data in 2011 based the image in 2009 with the GWR method, also can achieve the ideal visual effect which means the method has good fitness.

Compared with orthogonal regression correction, GWR correction can enhance the spatial texture information obviously. In contrast, the spectral information can be improved a little.

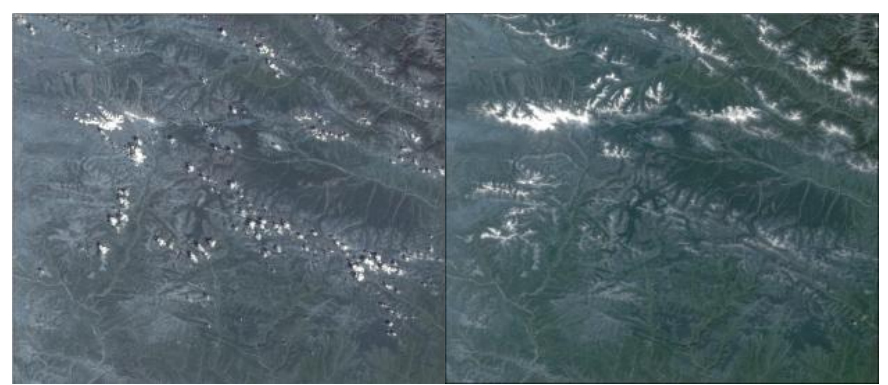

(a)The reference image of 2011 (b) The target image of 2009

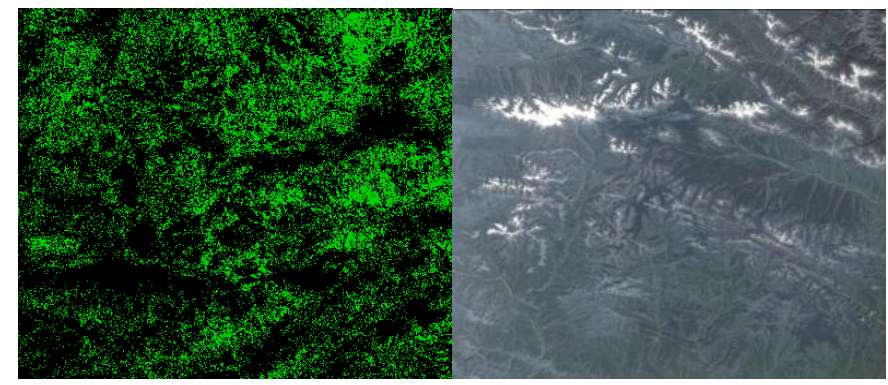

(c) The distribution of invariant points (d) The orthogonal regression correction results of 2009

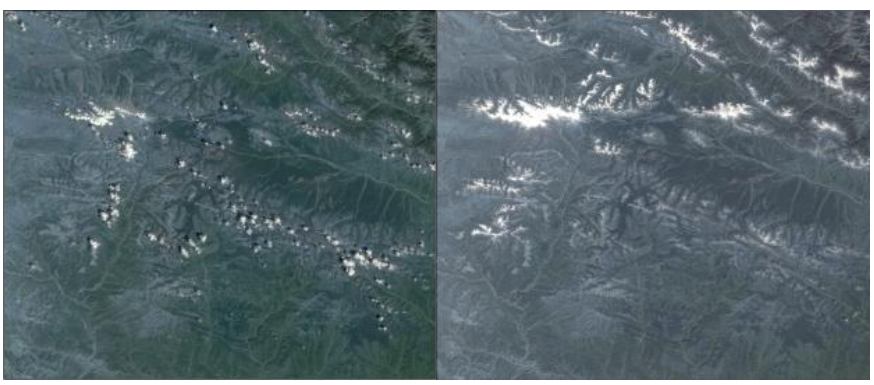

(e) GWR correction results of 2011 (f) GWR correction results of 2009

Fig. 1. Satellite images and radiometric correction results

It can be found from table 2 that the correlation coefficient of GWR radiometric correction results is larger than the orthogonal regression correction results, which means the result from GWR has higher correlation. Influenced by the low variation coefficient of reference image, the variation 
coefficients of GWR and orthogonal regression correction results are reduced, reducing the sparse degree of frequency distribution in original image. Information entropy of GWR radiometric correction results is larger than other images; it shows that the details of the image performance ability are enhanced greatly. A smaller deviation index means a smaller deviation degree between radiometric correction image and original image. From this point of view, the results of GWR radiometric correction is better.

TABLE II. THE LIST OF EVALUATION INDEX OF RADIOMETRIC CORRECTION RESULTS

\begin{tabular}{|c|c|c|c|c|c|}
\hline Band & Image & $\begin{array}{c}\text { Coefficient } \\
\text { of } \\
\text { correlation }\end{array}$ & $\begin{array}{c}\text { The } \\
\text { variation } \\
\text { coefficient }\end{array}$ & $\begin{array}{c}\text { Information } \\
\text { entropy }\end{array}$ & $\begin{array}{c}\text { Deviation } \\
\text { index }\end{array}$ \\
\hline \multirow{4}{*}{$\mathbf{R}$} & $\begin{array}{c}\text { reference } \\
\text { image }\end{array}$ & 1.0000 & 0.7716 & 3.4688 & 0.0510 \\
\hline & $\begin{array}{l}\text { target } \\
\text { image }\end{array}$ & 0.4528 & 1.3709 & 3.0538 & 0.0521 \\
\hline & $\begin{array}{c}\text { orthogonal } \\
\text { results }\end{array}$ & 0.4544 & 1.0759 & 3.0381 & 0.0290 \\
\hline & $\begin{array}{l}\text { GWR } \\
\text { results }\end{array}$ & 0.4566 & 1.0715 & 6.4514 & 0.0287 \\
\hline \multirow{4}{*}{ G } & $\begin{array}{c}\text { reference } \\
\text { image }\end{array}$ & 1.0000 & 0.6424 & 3.6891 & 0.0421 \\
\hline & $\begin{array}{l}\text { target } \\
\text { image }\end{array}$ & 0.4856 & 0.8959 & 3.1692 & 0.0425 \\
\hline & $\begin{array}{c}\text { orthogonal } \\
\text { results }\end{array}$ & 0.4857 & 0.8583 & 3.1546 & 0.0299 \\
\hline & $\begin{array}{l}\text { GWR } \\
\text { results }\end{array}$ & 0.4885 & 0.8586 & 7.4065 & 0.0296 \\
\hline \multirow{4}{*}{ B } & $\begin{array}{c}\text { reference } \\
\text { image }\end{array}$ & 1.0000 & 0.5398 & 3.9861 & 0.0519 \\
\hline & $\begin{array}{l}\text { target } \\
\text { image }\end{array}$ & 0.5544 & 0.8412 & 3.4879 & 0.0533 \\
\hline & $\begin{array}{c}\text { orthogonal } \\
\text { results }\end{array}$ & 0.5495 & 0.7091 & 3.4765 & 0.0282 \\
\hline & $\begin{array}{l}\text { GWR } \\
\text { results }\end{array}$ & 0.5516 & 0.7144 & 7.6198 & 0.0281 \\
\hline
\end{tabular}

\section{ANALYSIS AND DISCUSSION}

The relative radiometric correction method proposed in the paper takes the surrounding environment effects on surface radiation into consideration. The visual effect of this method has no obvious difference with that of the orthogonal regression radiometric correction. While the information entropy of the method improved greatly, which means the process can enhance the detail information of the origin image.

This paper presents the relative radiometric correction method relying on the spatial of invariant features points. Here non-uniform distribution will affect the visual effects of radiometric correction, which would induce piece effect in severe cases. It is also noteworthy, when handling large volume data it would be better to use the block index to find the closest samples to the target point. Our test has proved it can improve the processing efficiency greatly.

\section{REFERENCES}

[1] DaiX, Khorram S. The Effects of Image Misregistration on the Accuracy of Remotely Sensed Change Detection [J]. IEEE Transactions on Geoscience and Remote Sensing, 1998, 36 (9) :1566) 1577.

[2] B ruzzone L, Smits P C, Tilton J C. Foreword) Special Issue on Analysis of Multitemporal Remote Sensing Images [J]. IEEE Transactions on Geoscience and Remote Sensing, 2003, 41 (11):2419- 2422.

[3] F.G.Hall, D.E.Strebel, J.E.Nickeson, et al. Radiometric rectification: toward a common radiometric response among multidate, multisensor images [J].Remote Sensing. Environment, 1991, 35:11-27.

[4] Conghe Song, Curtis E.Woodcock, Karen C. Seto, et al. Classification and change detection using landsat TM data: When and how to correct atmospheric effects[J].Remote Sens.Environ.,2001,75:230-244.

[5] Guo Jianning, Yu Jin, Zeng Yong, et al. Relative Radiometric Correction for CBERS-01-02 CCD Images [J]. Science in China Ser.E Information Sciences,2005,35(Supplement I ):11-25.

[6] Mei Anxin, Peng Wanglu, Qin Qiming, et al. An Introduction to Remote Sensing [M]. Beijing: Higher Education Press. 2001.

[7] HALL F G, S TREBEL D E, NICKESON J E, et al. Radiometric Rectification: Toward a Common Radiomet ric Response among Multidata, Multi sensor Images [J]. Remote Sens Environ, 1991, 35: 11- 27.

[8] COPPINP R, BAUER M E. Digit al Change Detection in Forest Ecosystems with Remote Sensing Imagery [J]. Remote Sensing Review s, 1996, 13: 207- 234.

[9] Zhang Youshui, Feng Xuezhi, Zhou Chenghu. Relative Radiometric Correction for Multitemporal TM Images [J]. Acta Geodaetica ET Cartographica Sinica, 2006, 35(2):122-127.

[10] Zheng Yuquan, Cui Dunjie. Spectral Nonlinearity Correction for Ocean Color CCD Imager [J].Journal of Remote Sening,1999, 3(2):107-111.

[11] Wack E C. SWIR No n- linear Radiometr ic Response Due to Err or s in Relative Spectral Response. SPIE, 1997, 3 117: 217 224.

[12] Weinreb M. Nonlinearity Correction in Calibration of Advanced Very High Resolution Radiometer Infrared Channels. Journal of Geophysical Research, 1990, 95(C5)7381 7388.

[13] Fotheringham A S. Chris Brunsdon, Martin Charlton. Geographically Weighted Regression : The Analysis of Spatially Varying Relationships [M]. U. K. West Sussex : John Wiley \& SonsLtd, 2002.

[14] Xuan Haiyan, Li Suoping, Liu Shuqun. The Geographically Weighted Regression Model and Its Fit Methods [J].Journal of Gansu Sciences, 2007, 19(1):51-52.

[15] Wu Mei, Gu Saisai. The Statistical Inference of Variation Coefficient of Sample and Its Applications [J], Journal of Tongren University, 2010, 12(1):139-144.

[16] Xu Min, Liu Yong, Wang Xiaoyan, Qiao Rong. Comparison of Fusion Algorithms of IKONOSPAN and Multi-spectral Images [J]. Geospatial Information, 2011, 9(4):84-87.

[17] Canty, M. J. and Nielsen, A. A., 2008. Automatic radiometric normalization of multitemporal satellite imagery with the iteratively re-weighted MAD transformation Remote Sensing of Environment, 112(3): 1025-1036. 